\title{
Influência do transtorno fonológico sobre os transtornos de leitura e da escrita
}

\section{Influence of phonological disorders on reading and writing disabilities}

\author{
Monique Cantelli da Silva', Clara Regina Brandão de Ávila
}

\begin{abstract}
RESUMO
Objetivo: Investigar a influência do transtorno fonológico sobre o desempenho em tarefas de leitura e de escrita e as relações entre essas variáveis. Métodos: Foram avaliados 28 escolares matriculados no $4^{\circ}$ e $5^{\circ}$ anos do Ensino Fundamental, divididos em Grupo Pesquisa 1, Grupo Pesquisa 2 e Grupo Controle. Foram avaliadas as seguintes competências: leitura, escrita, processamento fonológico e auditivo. Os dados foram analisados estatisticamente. Resultados: O Grupo Controle mostrou melhores resultados em leitura, escrita e processamentos. Os Grupos Pesquisa mostraram-se semelhantes quanto à consciência fonológica e memória fonológica de curto prazo. O Grupo Pesquisa 1 mostrou valores médios de desempenho mais baixos na maioria das tarefas, quando comparado ao Grupo Pesquisa 2, embora sem diferença estatística. O processamento auditivo mostrou-se semelhante entre os três grupos, com exceção da prova de Memória Sequencial Verbal. Os três grupos apresentaram diferentes padrões de correlações entre as variáveis. Conclusão: O transtorno fonológico mostrou-se associado aos piores desempenhos em leitura, escrita e processamento fonológico. Os grupos apresentaram padrões diferentes de correlação entre as variáveis de leitura, escrita, auditiva, processamento auditivo e fonológico, o que confirmou os resultados das comparações.
\end{abstract}

Descritores: Distúrbios da fala; Transtornos de aprendizagem; Linguagem; Percepção auditiva; Fonoaudiologia

\begin{abstract}
Purpose: To investigate the influence of phonological disorder, present at school age on performance on tasks of reading, writing, phonological and auditory processing and the relationships between these variables. Methods: A total of 28 children regularly enrolled in $4^{\text {th }}$ and $5^{\text {th }}$ grade of elementary schools were divided: Experimental Group 1, Experimental Group 2, and Control Group. The following skills were assessed: reading, writing, phonological and auditory processing. The data were statistically analyzed. Results: Control Group showed better results in reading, writing and processings. Experimental Groups were similar in terms of phonological awareness and phonological short-term memory. Experimental Group 1 showed lower values of performances compared to Experimental 2, with or without statistical significance. Auditory processing was similar among three groups, with the exception of Sequential Verbal Memory task. The three groups showed different patterns of correlations between variables. Conclusion: The phonological disorder was associated with worse performance on reading, spelling and phonological processing. The different groups showed different patterns of correlations between reading, writing, listening and phonological processing, which corroborated the results of the comparisons.
\end{abstract}

Keywords: Speech disorders; Learning disorders; Language; Auditory perception; Speech, Language and Hearing Sciences

\footnotetext{
Trabalho realizado no Ambulatório de Fonoaudiologia do Hospital São Paulo e no Núcleo de Ensino, Assistência e Pesquisa em Escrita e Leitura (NEAPEL), Departamento de Fonoaudiologia, Universidade Federal de São Paulo - UNIFESP - São Paulo (SP), Brasil, com bolsa concedida pela Coordenação de Aperfeiçoamento de Pessoal de Nível Superior (CAPES).

(1) Programa de Pós-Graduação (Mestrado) em Distúrbios da Comunicação Humana, Universidade Federal de São Paulo - UNIFESP - São Paulo (SP), Brasil. (2) Departamento de Fonoaudiologia, Universidade Federal de São Paulo - UNIFESP - São Paulo (SP), Brasil.

Conflito de interesses: Não

Contribuição dos autores: $M C S$ pesquisadora principal, elaboração da pesquisa, elaboração do cronograma, levantamento da literatura, coleta e análise dos dados, redação do artigo, submissão e trâmites do artigo; CRBA orientadora, elaboração da pesquisa, análise dos dados, correção da redação do artigo, aprovação da versão final.

Endereço para correspondência: Monique Cantelli da Silva. R. Botucatu, 802, Vila Clementino, São Paulo (SP), Brasil, CEP: $04023-062$.

E-mail: moniquecantelli@gmail.com
}

Recebido em: 27/12/2012; Aceito em: 22/7/2013 


\section{INTRODUÇÃO}

Estudos sobre o desenvolvimento da linguagem identificam a existência de relação de continuidade entre as modalidades oral e escrita da comunicação humana ${ }^{(1-5)}$. Ambas compartilham funções e processamentos de diferentes subsistemas da linguagem, alguns dos quais - principalmente os fonológicos - são importantes para o aprendizado do princípio alfabético da escrita. As mesmas habilidades e informações linguísticas que, ao longo do desenvolvimento da linguagem oral e da fala, forneceram o suporte para o aprendizado de novos fonemas e vocábulos, são utilizadas de forma competente nas demandas perceptivas e metacognitivas para o aprendizado do sistema alfabético. Essas relações implicam e definem a necessidade da criançater desenvolvido, completa e adequadamente, o seu sistema de fala, ao ingressar no ensino fundamental.

Entretanto, estudos nacionais identificaram expressiva porcentagem de escolares que, a despeito de terem iniciado o processo de alfabetização, ainda apresentavam alterações de fala, caracterizadas, principalmente, pela presença de Transtorno Fonológico $(\mathrm{TF})^{(6-9)}$. Tendo em vista que as representações fonológicas são o substrato em que as informações formais sobre letras e palavras escritas serão sedimentadas - primordialmente a partir das habilidades de consciência fonológica e da associação fonema-grafema - poder-se-ia pensar, então, que o aprendizado da leitura e da escrita, na vigência da alteração de fala, não é tarefa fácil para o escolar.

Tanto a clínica quanto a pesquisa voltadas para os transtornos da leitura e da escrita têm relatado diferentes padrões de associação entre habilidades metafonológicas ou outras do processamento fonológico, com alterações que se manifestam na escrita e na leitura ${ }^{(10-12)}$, em função da presença ou ausência do transtorno de fala, em coocorrência.

Não raramente, escolares com diagnóstico de Transtorno da Leitura e/ou Transtorno da Escrita (TLE) apresentam, em suas histórias de desenvolvimento, atrasos ou alterações das aquisições fonêmicas. Outros evidenciam, na avaliação fonoaudiológica, o transtorno fonológico que, nessa etapa, deve agravar os prejuízos expressos na leitura e/ou na escrita. Nesse caso, o escolar será duplamente penalizado em seu esforço para ler e escrever.

Entre os escolares com TLE, há aqueles que, não obstante a faixa etária, ainda apresentam alterações persistentes de fala, sobretudo fonológicas, ou fonético-fonológicas. Segundo a literatura, essas alterações podem ser assim definidas quando presentes em crianças na idade escolar, tipicamente, a partir do período compreendido entre cinco e seis anos, ou que permanecem para além dos 9 anos de idade ${ }^{(1,2,13)}$. Nessas condições, observa-se desde transtornos de fala já tratados, ou padrões de fala que se normalizaram tardiamente, mesmo sem a intervenção fonoaudiológica, até a persistência de dificuldades da fala durante a escolarização e que podem continuar até a vida adulta, em crianças que não responderam satisfatoriamente à intervenção terapêutica.

As correlações entre a presença de alterações fonológicas e déficits de processamento fonológico são conhecidas ${ }^{(9,14,15)}$. Estes últimos, certamente, aumentam o risco do surgimento de dificuldades no aprendizado do princípio alfabético, prejudicando o próprio aprendizado e uso da leitura e da escrita ${ }^{(10,11,13,16,17)}$, ou do agravamento das alterações já instaladas.

Assim, a hipótese de que o TF manifesto na fala, ainda presente na idade escolar, possa dificultar, agravar ou piorar as condições de aprendizado da leitura e da escrita conduziu esta pesquisa. Admitindo que diferentes capacidades de processamento de informações linguísticas, fonológicas, etc. subjazem o aprendizado do ler e escrever, as piores ou melhores condições de leitura e de escrita podem ser caracterizadas, também, no nível desses processamentos e, da mesma forma, mostrar diferentes níveis de habilidades caracterizando diferentes quadros ou subtipos. Portanto, também foi levantada a hipótese de que os diferentes desempenhos em processamento fonológico, auditivo, leitura e escrita possam determinar diferentes padrões de correlação, na presença ou não do TF quando o TLE se apresenta.

Por isso, o objetivo desta pesquisa foi investigar a influência do TF, ainda presente na idade escolar, sobre o desempenho em tarefas de leitura, escrita e dos processamentos fonológico e auditivo, além de investigar possíveis relações entre essas variáveis.

\section{MÉTODOS}

Pesquisa aprovada pelo Comitê de Ética em Pesquisa da Universidade Federal de São Paulo (UNIFESP) - Hospital São Paulo, sob número 0438/10. Teve início após a anuência das escolas e assinaturas do Termo de Consentimento Livre e Esclarecido (TCLE) pelos pais dos escolares. A coleta dos dados ocorreu no primeiro semestre letivo de 2011, durante um período de três meses.

De um total de 51 escolares inicialmente triados, 28 (oito meninas), com idades entre 9 e 12 anos, compuseram a amostra. Embora não se tenha considerado o nível socioeconômico dos participantes para a constituição da amostra, todos estavam regularmente matriculados na rede pública de ensino dos municípios de São Paulo e Embu das Artes.

Os critérios de inclusão na amostra foram: ausência de queixas ou indicadores da presença de alterações ou déficits da audição, da visão (não corrigidos) e de distúrbios neurológicos, comportamentais ou cognitivos; história de retenção escolar; realização da triagem fonoaudiológica para confirmação das características a serem observadas para a composição da amostra. Foram excluídos da amostra os escolares que não se mostraram, ao menos, na condição silábico-alfabética de escrita ${ }^{(18)}$ de palavras de alta frequência, ou que não liam palavras, ainda que de forma silabada e os escolares que não apresentaram o 
reflexo cócleo-palpebral (RCP) na Avaliação Simplificada do Processamento Auditivo ${ }^{(19)}$.

Os escolares foram recrutados ao chegarem aos Ambulatórios do Departamento de Fonoaudiologia do Hospital São Paulo, com queixa relacionada à dificuldade de aprendizado, ou nas escolas da rede pública de ensino, que participaram da pesquisa. Foram triados para a composição de três grupos delineados, $a$ priori: Grupo Controle (GC): escolares considerados de bom rendimento escolar, por seus professores ${ }^{(20)}$ e sem relato de retenção escolar; Grupo de Pesquisa 1 (GP1): escolares com queixa ou indícios de alterações relacionadas ao aprendizado da leitura e da escrita e de alterações de fala; Grupo de Pesquisa 2 (GP2): escolares com queixa ou indícios de alterações relacionadas ao aprendizado da leitura e da escrita e sem queixas relacionadas à fala.

Passaram por avaliação fonoaudiológica para confirmação da presença ou ausência de alterações de leitura, escrita e de fala e formulação dos diagnósticos fonoaudiológicos, quando pertinentes. Os resultados obtidos nessa avaliação foram utilizados, primeiramente, para a seleção da amostra e estruturação dos grupos participantes. Esses mesmos resultados, juntamente com os obtidos nas outras avaliações, compuseram o total de dados sobre a leitura, a escrita, a fala, o processamento fonológico e o auditivo, que permitiram a realização de comparações entre os grupos e a investigação de correlações entre as variáveis.

No que diz respeito à comunicação escrita, este estudo investigou apenas capacidades relacionadas à codificação e à decodificação. Aplicaram-se provas de Leitura oral de itens isolados - duas listas (38 palavras e 29 pseudopalavras), balanceadas quanto à extensão, frequência e ortografia ${ }^{(20,21)} \mathrm{e} \mathrm{Leitura}$ oral de texto adequado à escolaridade ${ }^{(22)}$, calculando-se as taxas e as acurácias dessas leituras. Os valores encontrados foram comparados com os descritos por outros autores ${ }^{(20)}$, por série escolar, para verificação de déficits de decodificação leitora e para a exclusão da amostra, quando indicados. Aplicou-se também as provas Escrita sob ditado de duas listas (35 palavras e 21 pseudopalavras) ${ }^{(21,23)}$ e Escrita autônoma de história a partir de figuras em sequência. Como procedimento para exclusão da amostra, as produções escritas foram classificadas, segundo suas características, como expressivas de uma das quatro hipóteses de escrita ${ }^{(18)}$ : pré-silábica, silábica, silábico-alfabética e alfabética. Além disso, computou-se o número de palavras e pseudopalavras escritas corretamente, para a comparação entre os grupos.

Para a avaliação da comunicação oral aplicou-se a prova de Fonologia do Teste de Linguagem Infantil - ABFW ${ }^{(24)}$. Deste protocolo, utilizou-se apenas a nomeação de figuras. Identificou-se e analisou-se os processos fonológicos na fala dos escolares, quando presentes, e calculou-se as produtividades desses processos, caracterizando a presença de TF e definindo o agrupamento dos escolares com TLE nos dois grupos de pesquisa. Compuseram o GP1 apenas os escolares que apresentaram, pelo menos, um processo fonológico produtivo.
Os 28 escolares foram, então, agrupados em: GP1: sete escolares com diagnóstico fonoaudiológico de TLE e TF; GP2: oito escolares com diagnóstico fonoaudiológico de TLE (sem evidências de Transtorno Fonológico); GC: 13 escolares sem TLE ou TF, ou queixas relacionadas, indicados pelos professores por apresentarem bom rendimento escolar.

Todos foram avaliados, também, em suas habilidades de processar informações fonológicas e auditivas, por meio dos seguintes protocolos:

1. Avaliação Simplificada do Processamento Auditivo (ASPA)

(19) - Provas: localização da fonte sonora em cinco direções; memória sequencial não verbal (MSNV); memória sequencial verbal (MSV);

2. Avaliação do Processamento Fonológico: Teste de Memória Fonológica de Trabalho - Brazilian Pseudoword Repetition (BCPR) ${ }^{(25)}$; Teste de Nomeação Rápida (Objetos e Cores) - Comprehensive Test of Phonological Processing $(\mathrm{CTOPP})^{(26)}$; Prova de Consciência Fonológica (PCF) ${ }^{(27)}$.

Aplicou-se o teste não paramétrico Kruskal-Wallis, visando comparar os dados obtidos entre os grupos estudados e o Grupo Controle. Quando detectadas diferenças, foram utilizadas as Comparações Múltiplas de Tukey. O coeficiente de correlação de Spearman foi calculado para investigar as possíveis relações entre as variáveis de leitura e escrita e de processamento auditivo e fonológico, nos três grupos. Para todos os testes fixou-se em 0,05 o nível de significância.

\section{RESULTADOS}

O GC mostrou médias mais elevadas de taxa e de acurácia na leitura de itens isolados ou de texto e, em média, acertou mais ao escrever sob ditado, quando seu desempenho foi comparado ao do GP1 e do GP2. Apesar do GP1 ter apresentado valores mais baixos de média de itens lidos e escritos corretamente, ambos os grupos pesquisa mostraram desempenho estatisticamente semelhante nas tarefas de leitura e escrita (Tabela 1).

A comparação entre os três grupos mostrou maiores médias de acerto do GC nas tarefas que avaliaram consciência fonológica. Os dois grupos pesquisa apresentaram desempenhos semelhantes nessas tarefas. A avaliação da memória fonológica de curto prazo mostrou maiores valores médios do GC, quando comparados aos do GP1, para a maioria dos estímulos, não ocorrendo diferença com relação ao GP2, nessa prova. Entretanto, quando os estímulos foram de alta similaridade com palavras, os desempenhos entre os três grupos foram semelhantes. O tempo de nomeação rápida de objetos mostrou que o GC e o GP2 foram diferentes, com médias mais baixas do GC. Contudo, não houve diferença entre GC e GP1 e entre GP1 e GP2 quanto ao tempo despendido para nomear objetos. A média de erros observados durante a nomeação de objetos e de cores e o tempo despendido na nomeação de cores não diferiu entre os três grupos (Tabela 1). 
Tabela 1. Comparação das médias de acerto nas avaliaç̃oes de leitura, escrita e processamentos fonológico e auditivo de GP1, GP2 e GC

\begin{tabular}{|c|c|c|c|c|c|c|c|}
\hline & & & Grupo & & & & \\
\hline & & $\begin{array}{c}\mathrm{GC} \\
\mathrm{n}=13\end{array}$ & $\begin{array}{l}\text { GP1 } \\
n=7\end{array}$ & $\begin{array}{l}\text { GP2 } \\
n=8\end{array}$ & Valor de $p$ & de Tukey & Resultado \\
\hline Taxa de palavras & Mediana & 35,1 & 4,7 & 15,9 & $<0,001^{*}$ & GCxGP2 $(p)<0,001^{*}$ & $\mathrm{GC}>\mathrm{GP} 1=\mathrm{GP} 2$ \\
\hline & DP & 10,8 & 5,6 & 12,9 & & GP1xGP2 $(p)=0,119$ & \\
\hline & DP & 7,0 & 5,0 & 10,9 & & GP1xGP2 $(p)=0,056$ & \\
\hline & Média & 71,5 & 9,9 & 27,8 & & GCxGP1 $(p)<0,001^{*}$ & \\
\hline Taxa de texto & Mediana & 69,9 & 9,2 & 17,8 & $<0,001^{*}$ & GCxGP2 $(p)<0,001^{*}$ & $\mathrm{GC}>\mathrm{GP} 1=\mathrm{GP} 2$ \\
\hline & DP & 18,8 & 8,7 & 26,7 & & GP1xGP2 $(p)=0,203$ & \\
\hline & Média & 21,4 & 1,0 & 6,5 & & GCxGP1 $(p)<0,001^{*}$ & \\
\hline Acurácia de & Mediana & 20,6 & 0,0 & 4,5 & $<0,001^{*}$ & GCxGP2 $(p)<0,001^{*}$ & $\mathrm{GC}>\mathrm{GP} 1=\mathrm{GP} 2$ \\
\hline & DP & 6,9 & 2,3 & 7,1 & & GP1xGP2 $(p)=0,217$ & \\
\hline & Média & 66,4 & 4,0 & 22,4 & & $\operatorname{GCxGP1}(p)<0,001^{*}$ & \\
\hline Acurácia de texto & Mediana & 66,3 & 1,9 & 10,4 & $<0,001^{*}$ & GCxGP2 $(p)<0,001^{*}$ & $\mathrm{GC}>\mathrm{GP} 1=\mathrm{GP} 2$ \\
\hline & DP & 20,7 & 6,0 & 26,8 & & $\operatorname{GP} 1 x G P 2(p)=0,210$ & \\
\hline & Média & 53,8 & 10,3 & 18,9 & & GCxGP1 $(p)<0,001^{*}$ & \\
\hline Acertos do ditado de & Mediana & 51,4 & 11,8 & 17,1 & $<0,001^{*}$ & GCxGP2 $(p)<0,001^{*}$ & $\mathrm{GC}>\mathrm{GP} 1=\mathrm{GP} 2$ \\
\hline & DP & 12,6 & 7,4 & 17,8 & & GP1xGP2 $(p)=0,439$ & \\
\hline & Média & 9,0 & 5,4 & 7,4 & & $\operatorname{GCxGP1}(p)=0,001^{*}$ & \\
\hline $\begin{array}{l}\text { Memoria de trabalho - } \\
\text { baixa similaridade }\end{array}$ & Mediana & 9,0 & 6,0 & 8,0 & $0,003^{*}$ & $\operatorname{GCxGP2}(p)=0,133$ & $\mathrm{GC}>\mathrm{GP} 1$ \\
\hline & DP & 1,0 & 1,9 & 2,6 & & GP1xGP2 $(p)=0,114$ & \\
\hline & Média & 17,5 & 11,6 & 14,6 & & $\operatorname{GCxGP1}(p)=0,002^{*}$ & \\
\hline $\begin{array}{l}\text { Memória de trabalho - } \\
\text { média similaridade }\end{array}$ & Mediana & 18,0 & 13,0 & 15,0 & $0,007^{*}$ & $\operatorname{GCxGP2}(p)=0,137$ & $\mathrm{GC}>\mathrm{GP} 1$ \\
\hline & DP & 1,8 & 4,1 & 4,2 & & $\operatorname{GP} 1 \times G P 2(p)=0,188$ & \\
\hline & Média & 9,5 & 8,4 & 9,3 & & & \\
\hline Memoria de trabalho - alta & Mediana & 10,0 & 9,0 & 9,5 & 0,132 & ------------------- & $\mathrm{GC}=\mathrm{GP} 1=\mathrm{GP} 2$ \\
\hline & DP & 1,2 & 1,5 & 0,9 & & & \\
\hline & Média & 36,0 & 25,4 & 31,3 & & $\operatorname{GCxGP1}(p)=0,001^{*}$ & \\
\hline Memória de trabalho - & Mediana & 37,0 & 26,0 & 32,5 & $0,003^{*}$ & $\operatorname{GCxGP2}(p)=0,129$ & $\mathrm{GC}>\mathrm{GP} 1$ \\
\hline & DP & 3,0 & 6,0 & 7,2 & & $\operatorname{GP} 1 x G P 2(p)=0,101$ & \\
\hline & Média & 72,1 & 98,1 & 101,9 & & $\operatorname{GCxGP1}(p)=0,100$ & \\
\hline $\begin{array}{l}\text { Nomeaçao rapida - tempo } \\
\text { obietos }\end{array}$ & Mediana & 70,0 & 92,0 & 94,0 & $0,006^{*}$ & GCxGP2 $(p)=0,043^{*}$ & $\mathrm{GC}>\mathrm{GP} 2$ \\
\hline & DP & 16,7 & 25,5 & 36,9 & & $\operatorname{GP} 1 \times G P 2(p)=0,958$ & \\
\hline & Média & 4,2 & 5,6 & 7,1 & & & \\
\hline $\begin{array}{l}\text { Nomeação rápida - erros } \\
\text { obietos }\end{array}$ & Mediana & 4,0 & 5,0 & 7,0 & 0,181 & -------------------- & $\mathrm{GC}=\mathrm{GP} 1=\mathrm{GP} 2$ \\
\hline
\end{tabular}


Tabela 1. continuação

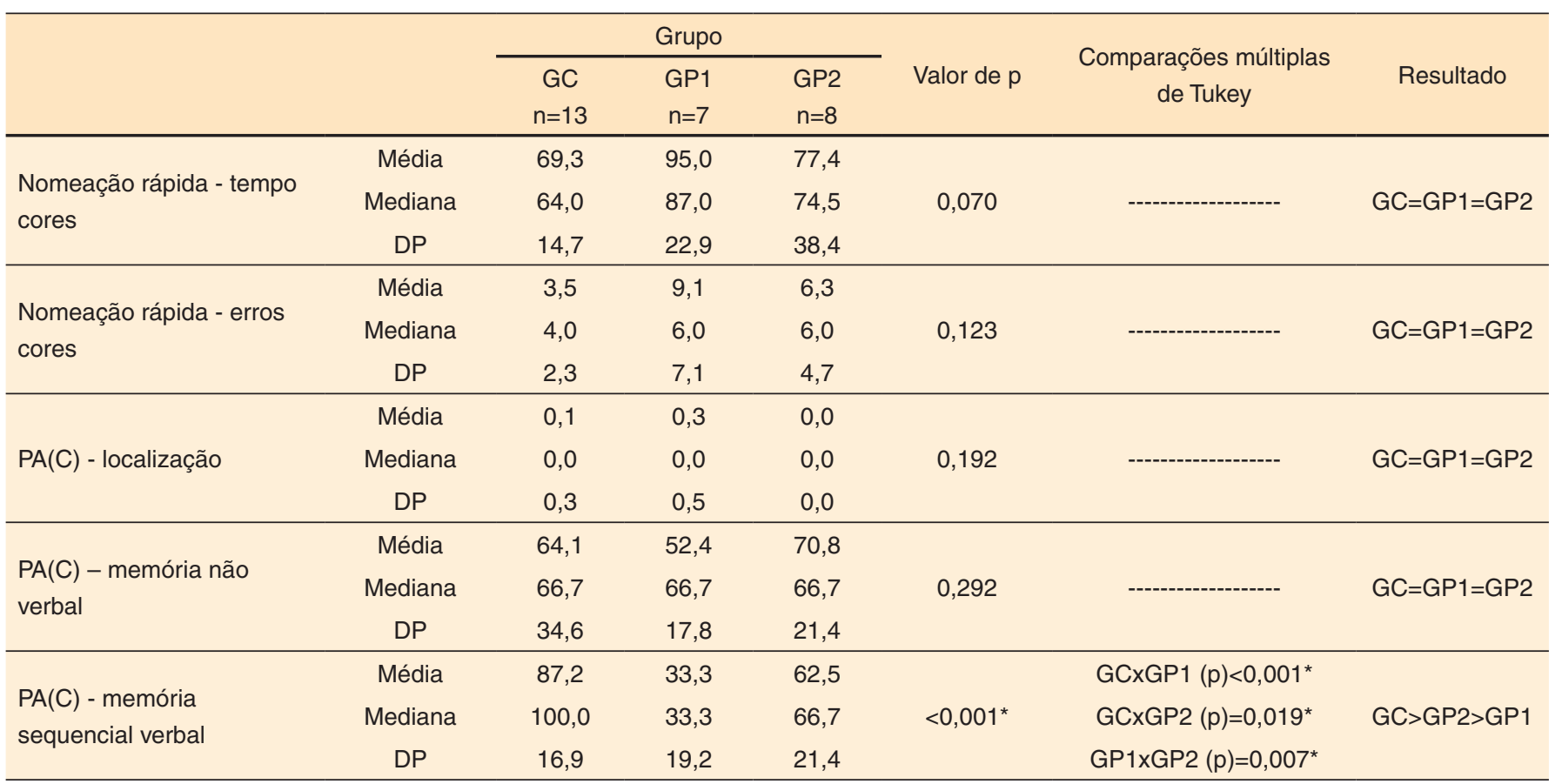

*Valores significativos $(p \leq 0,05)$ - teste de Kruskal-Wallis

Legenda: GC = Grupo Controle; GP1 = Grupo Pesquisa 1; GP2 = Grupo Pesquisa 2; DP = desvio-padrão; PA(C) = Processamento Auditivo (Central)

Os três grupos mostraram-se semelhantes quanto à localização da fonte sonora e da memória sequencial não verbal. Entretanto, quando comparada, as médias apresentadas pelo GC foram mais altas que as de GP2 e as deste grupo, mais altas que as do GP1 (Tabela 1).

A investigação das correlações entre as variáveis de leitura e escrita e as demais de processamentos fonológico e auditivo constatou correlações positivas para o GC, variando de moderadas a ótimas. As correlações encontradas ficaram circunscritas às variáveis de mesma natureza, isto é, as variáveis de leitura e escrita correlacionaram-se entre si, as de memória fonológica de trabalho entre si, assim como as de nomeação rápida (Tabela 2).

Não foram observadas, nesse grupo, correlações entre memória fonológica e variáveis de leitura e escrita. Por outro lado, a consciência fonológica correlacionou-se positivamente com o número de acertos do ditado de pseudopalavras (Tabela 2).

As análises do GP1 mostraram correlações que variaram de boas a ótimas. Entretanto, nem todas as provas de leitura correlacionaram-se entre si e nem com as de escrita. Encontraram-se diferentes correlações entre processamento fonológico e variáveis de leitura e de escrita. Assim, taxas de palavras, de pseudopalavras ou de texto, nas tarefas de leitura, correlacionaram-se positivamente, bem como a acurácia na leitura de texto e a taxa de leitura de palavras e de texto. Também foi encontrada correlação negativa entre o número de palavras escritas corretamente, sob ditado, e o tempo despendido na nomeação rápida de objetos. Já entre consciência fonológica e taxa e acurácia nas leituras (itens isolados e texto) e escrita correta de palavras, a correlação foi positiva. As memórias de trabalho para baixa e média similaridade correlacionaram-se positivamente com o escore total da memória fonológica, assim como o tempo e os erros de nomeação rápida de objetos com o tempo despendido na nomeação de cores. Também houve correlação negativa entre os tempos da nomeação rápida (cores e objetos) com a memória sequencial verbal (Tabela 3).

O GP2 mostrou o maior número de correlações. As variáveis de leitura e escrita correlacionaram-se entre si, nesse grupo. A memória fonológica de trabalho correlacionou-se positivamente com todas as variáveis de leitura e de escrita. Observou-se, também, que a memória de trabalho para pseudopalavras de baixa e média similaridade correlacionou-se positivamente com o escore total dessa prova. Além disso, verificou-se correlação negativa entre a memória e a nomeação rápida de objetos (Tabela 4).

Quanto às habilidades do processamento auditivo, o GP2 mostrou correlação da memória sequencial verbal com a taxa de leitura de texto, as acurácias de leitura, o ditado de palavras e os erros de nomeação rápida de objetos (Tabela 4).

\section{DISCUSSÃO}

Esta pesquisa investigou a hipótese da existência de subjacências fonológicas compartilhadas entre processamentos de fala e a alfabetização.

O estudo mostrou médias de acertos mais elevadas do GC em todas as variáveis de leitura e escrita e de consciência fonológica (Tabela 1), significando que esses valores foram compatíveis com os indicados na literatura para escolares sem queixa ${ }^{(20,21,27)}$. Desta forma, esse grupo se mostrou adequado para constituir-se como baliza das comparações e correlações 
Tabela 2. Correlações entre as variáveis avaliadas em GC

\begin{tabular}{|c|c|c|c|c|c|c|c|c|c|c|c|c|c|c|c|c|c|c|c|c|}
\hline & 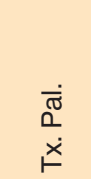 & $\underset{\dot{\alpha}}{\dot{x}}$ & 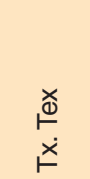 & $\begin{array}{l}\dot{\sigma} \\
\text { வ } \\
\dot{0}\end{array}$ & $\begin{array}{l}\dot{Q} \\
\dot{0} \\
\dot{\alpha}\end{array}$ & 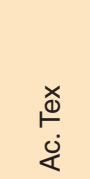 & $\begin{array}{l}\frac{\pi}{ \pm} \\
\frac{1}{0} \\
\frac{0}{\sigma} \\
0 \\
+\dot{0}\end{array}$ & 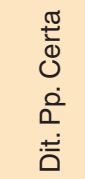 & $\begin{array}{l}\infty \\
\infty \\
1 \\
\leftarrow \\
\stackrel{\leftarrow}{\Sigma}\end{array}$ & $\begin{array}{c}\sum_{1}^{\infty} \\
5 \\
\frac{5}{\Sigma}\end{array}$ & $\begin{array}{l}0 \\
1 \\
1 \\
5 \\
\\
\Sigma\end{array}$ & 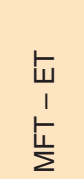 & $\begin{array}{l}\stackrel{\varrho}{E} \\
\frac{1}{\Phi} \\
: \frac{1}{0} \\
0 \\
\frac{\Upsilon}{Z}\end{array}$ & $\begin{array}{l}\frac{0}{\frac{2}{0}} \\
\frac{1}{O} \\
\frac{1}{0} \\
\frac{\Upsilon 1}{Z}\end{array}$ & 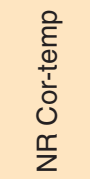 & 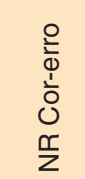 & U & 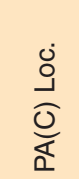 & 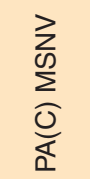 & $\frac{\sum_{0}^{\infty}}{0}$ \\
\hline Tx. Pal. & 1 & & & & & & & & & & & & & & & & & & & \\
\hline Tx. Pp. &, $803^{\star *}$ & 1 & & & & & & & & & & & & & & & & & & \\
\hline Ac. Pal. &, $989^{\star \star}$ &, $779^{\star *}$ &, $725^{\star \star}$ & 1 & & & & & & & & & & & & & & & & \\
\hline Ac. Pp. &, $771^{* *}$ &, $762^{* *}$ &, $560^{\star}$ &, $742^{* *}$ & 1 & & & & & & & & & & & & & & & \\
\hline Ac. Tex & ,678* &, $561^{*}$ &, $978^{\star \star}$ &, $692^{\star *}$ &, $566^{\star}$ & 1 & & & & & & & & & & & & & & \\
\hline Dit. Pal acert & 0,418 & 0,261 &, $621^{*}$ & 0,463 & 0,439 &, $698^{\star *}$ & 1 & & & & & & & & & & & & & \\
\hline MFT-AS & $-0,471$ & $-0,259$ & $-0,323$ & $-0,49$ & $-0,327$ & $-0,323$ & $3-0,095$ & $-0,351$ & 0,162 & 0,247 & 1 & & & & & & & & & \\
\hline MST-ET & $-0,006$ & 0,017 & $-0,105$ & 0,031 & $-0,096$ & $-0,127$ & $7-0,02$ & 0,255 & ,651* &, $860^{* *}$ & 0,501 & 1 & & & & & & & & \\
\hline NR Obj- T & $-0,419$ & $-0,275$ & $-0,074$ & $-0,377$ & $-0,143$ & $-0,151$ & $-0,083$ & $-0,126$ & $-0,328$ & 0,025 & 0,324 & 0,031 & 1 & & & & & & & \\
\hline NR Obj-Er & $-0,407$ & $-0,284$ & $-0,045$ & $-0,359$ & $-0,157$ & $-0,132$ & $-0,175$ & $-0,021$ & $-0,239$ & 0,138 & 0,247 & 0,117 &, $947^{\star *}$ & 1 & & & & & & \\
\hline NRCor-T & $-0,281$ & 0,039 & $-0,088$ & $-0,231$ & $-0,044$ & $-0,137$ & 0,083 & 0 & $-0,089$ & 0,078 & 0,401 & 0,224 &, $834^{\star \star}$ &, $727^{\star \star}$ & 1 & & & & & \\
\hline NR Cor-Er & $-0,51$ & $-0,207$ & $-0,318$ & $-0,465$ & $-0,265$ & $-0,363$ & $3-0,19$ & $-0,269$ & $-0,004$ & $-0,022$ &, $572^{*}$ & 0,2 &, $830^{\star *}$ &, $735^{\star \star}$ &, $874^{\star \star}$ & 1 & & & & \\
\hline CF & 0,535 & 0,351 &, $829^{\star \star}$ &, $558^{\star}$ & 0,497 &, $785^{\star *}$ & * 0,48 &, $767^{\star *}$ & $-0,067$ & 0,423 & $-0,262$ & 0,182 & $-0,039$ & 0,087 & $-0,16$ & $-0,39$ & 1 & & & \\
\hline PA(C) Loc. & $-0,116$ & 0 & $-0,154$ & $-0,154$ & $-0,154$ & $-0,077$ & 70,194 & $-0,275$ & $-0,324$ & $-0,285$ & 0,157 & $-0,238$ & 0 & $-0,276$ & 0,231 & 0,119 & $-0,47$ & 1 & & \\
\hline $\mathrm{PA}(\mathrm{C}) \mathrm{MSNV}$ & 0,024 & $-0,015$ & 0,447 & 0,082 & $-0,088$ & 0,535 & 0,517 &, $566^{*}$ & 0,088 & $-0,29$ & $-0,4$ & $-0,203$ & $-0,212$ & $-0,141$ & $-0,162$ & $-0,28$ & 0,358 & $-0,04$ & 1 & \\
\hline
\end{tabular}

*Valores significativos $(p \leq 0,05)$ - coeficiente de Correlação de Spearman; **Valores significativos $(p \leq 0,01)$ - coeficiente de Correlação de Spearman

Legenda: Tx. Pal. = taxa de palavras; Tx. Pp. = taxa de pseudopalavras; Tx. Texto = taxa de texto; Ac. Pal. = acurácia de palavras; Ac. Pp. = acurácia de pseudopalavras; Ac. Texto = acurácia de texto; MFT-BS = memória fonológica de trabalho com palavras de baixa similaridade; MFT-MS = memória fonológica de trabalho com palavras de média similaridade; MFT-AS = memória fonológica de trabalho com palavras de alta similaridade; MFT-ET = memória fonológica de trabalho - escore total; NR Obj.T = tempo de nomeação rápida de objetos; NR Cor.T = tempo de nomeação rápida de cores; NR Obj Er = erros de nomeação rápida de objetos; NR Cor $\mathrm{Er}$ = erros de nomeação rápida de cores; $\mathrm{CF}=$ consciência fonológica; $\mathrm{PA}(\mathrm{C})$ - Loc. = localização da fonte sonora; $\mathrm{PA}(\mathrm{C})-\mathrm{MSNV}=$ memória sequencial não verbal; $\mathrm{PA}(\mathrm{C})-\mathrm{MSV}=$ memória sequencial verbal

realizadas com os demais grupos de pesquisa.

Os três grupos mostraram desempenho em consciência fonológica compatível com as características identificadas na avaliação da leitura e escrita. Isto é, o GC apresentou melhor desempenho que o GP2 e este, apesar da ausência de significância estatística, acertou mais que o GP1, no geral, confirmando as relações existentes entre o processamento das informações fonológicas e as condições de escrita e leitura do escolar(5,16).

Por outro lado, deve-se ressaltar que o GP2 apresentou resultados semelhantes ao GC na avaliação da memória fonológica de trabalho. As tarefas de memória fonológica são particularmente difíceis para crianças com problemas de fala que persistiram além do início da alfabetização, como os escolares do GP1, que também mostraram os mais baixos valores médios em leitura e em escrita ${ }^{(2,13)}$. Esses resultados estão de acordo com a literatura, segundo a qual, os problemas de fala interferem nos resultados do aprendizado do princípio alfabético, quanto mais graves, ou quanto mais duradoura for a persistência das alterações fonológicas ${ }^{(2,8,9,17)}$.

Considerando-se os resultados descritos na Avaliação Simplificada do Processamento Auditivo (Tabela 1), pode-se concluir que os escolares com Transtorno Fonológico (GP1) mostraram pior desempenho em todas as comparações realizadas entre os grupos e admitir a possibilidade de, por esse motivo, ser pior, também, seu desempenho em leitura e escrita $^{(2,8,9,17)}$. As análises por comparação puderam mostrar que os três grupos são, de alguma forma, diferentes e que essa diferença foi observada, principalmente, pelas respostas do GC, que se mostraram dentro do esperado para a normalidade; pelas respostas dos grupos pesquisa, que mostraram piores desempenhos em leitura, escrita e consciência fonológica e pelo pior desempenho do grupo de escolares com TLE e TF (GP1) em relação aos demais, quando se considerou o total das provas aplicadas na avaliação fonoaudiológica. O pior desempenho em memória fonológica de trabalho pode ter caracterizado

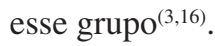


Tabela 3. Correlações entre as variáveis avaliadas em GP1

\begin{tabular}{|c|c|c|c|c|c|c|c|c|c|c|c|c|c|c|c|c|c|c|c|c|}
\hline & $\begin{array}{l}\dot{\sigma} \\
\dot{\alpha} \\
\dot{x}\end{array}$ & $\begin{array}{l}\dot{Q} \\
\dot{x} \\
\dot{x}\end{array}$ & 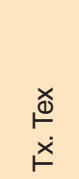 & $\begin{array}{l}\dot{\sigma} \\
\dot{0} \\
\dot{0}\end{array}$ & $\frac{\dot{0}}{\dot{0}}$ & $\begin{array}{l}\stackrel{x}{\oplus} \\
\stackrel{0}{<}\end{array}$ & 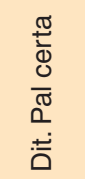 & $\begin{array}{l}\frac{\pi}{\frac{\pi}{0}} \\
0 \\
0 \\
\dot{0} \\
\dot{0} \\
+\frac{1}{0}\end{array}$ & 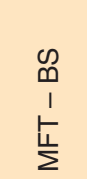 & $\begin{array}{l}\sum_{1}^{\infty} \\
\frac{1}{\Sigma} \\
\frac{1}{\Sigma}\end{array}$ & $\begin{array}{c}0 \\
1 \\
1 \\
5 \\
\\
\Sigma\end{array}$ & $\begin{array}{c}5 \\
\mathbf{1} \\
\leftarrow \\
\stackrel{5}{\Sigma}\end{array}$ & $\begin{array}{l}\stackrel{\varrho}{E} \\
\frac{d}{\Phi} \\
: \frac{1}{0} \\
0 \\
\frac{\Upsilon}{Z}\end{array}$ & $\begin{array}{l}\frac{O}{\frac{1}{0}} \\
\frac{1}{0} \\
0 \\
0 \\
\frac{\Upsilon}{Z}\end{array}$ & $\begin{array}{l}\stackrel{\circ}{E} \\
\frac{1}{ \pm} \\
\frac{1}{0} \\
\mathcal{O} \\
\frac{\Upsilon}{Z}\end{array}$ & 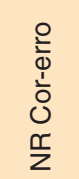 & U & 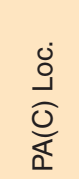 & 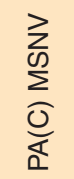 & $\frac{\sum_{0}^{\infty}}{0}$ \\
\hline Tx. Pal. & 1 & & & & & & & & & & & & & & & & & & & \\
\hline Tx. Pp. &, $964^{\star *}$ & 1 & & & & & & & & & & & & & & & & & & \\
\hline Ac. Pal. & 0,445 & 0,296 & 0,371 & 1 & & & & & & & & & & & & & & & & \\
\hline Ac. Pp. &, $802^{*}$ & 0,668 & 0,668 &, $832^{*}$ & 1 & & & & & & & & & & & & & & & \\
\hline Ac. Tex &, $786^{*}$ & 0,75 &, $857^{*}$ & 0,519 & 0,668 & 1 & & & & & & & & & & & & & & \\
\hline Dit. Pal acert & 0,487 & 0,523 & 0,595 & 0,168 & 0,225 &, $811^{*}$ & 1 & & & & & & & & & & & & & \\
\hline MFT-AS & 0 & 0,2 & 0,145 & $-0,66$ & $-0,318$ & $-0,109$ & 0,009 & 0,03 & 0,296 & $-0,111$ & 1 & & & & & & & & & \\
\hline MST-ET & 0,252 & 0,396 & 0,27 & $-0,168$ & $-0,045$ & 0,162 & 0,536 & 0,121 &, $908^{* *}$ & ,844* & 0,385 & 1 & & & & & & & & \\
\hline NR Obj- T & $-0,286$ & $-0,321$ & $-0,357$ & 0,296 & 0,178 & $-0,5$ &,$- 775^{\star}$ & 0,06 & $-0,291$ & $-0,273$ & $-0,036$ & $-0,324$ & 1 & & & & & & & \\
\hline NR Obj-Er & $-0,371$ & $-0,445$ & $-0,445$ & 0,385 & 0,185 & $-0,445$ & $-0,692$ & $-0,062$ & $-0,415$ & $-0,226$ & $-0,17$ & $-0,393$ &, $964^{\star \star}$ & 1 & & & & & & \\
\hline NRCor-T & $-0,393$ & $-0,429$ & $-0,536$ & 0,148 & 0,045 & $-0,607$ & $-0,667$ & $-0,179$ & $-0,055$ & 0,018 & 0,018 & $-0,036$ &, $893^{\star *}$ &, $889^{\star *}$ & 1 & & & & & \\
\hline NR Cor-Er & $-0,179$ & $-0,036$ & 0 & $-0,074$ & $-0,134$ & $-0,357$ & $-0,559$ & 0,239 & 0 & $-0,418$ & 0,346 & $-0,18$ & 0,607 & 0,445 & 0,321 & 1 & & & & \\
\hline CF &, $793^{*}$ &, $847^{*}$ &, $937^{* *}$ & 0,337 & 0,539 &, $937^{\star *}$ & ,800* & 0,724 & 0,128 & 0,321 & 0,156 & 0,336 & $-0,505$ & $-0,524$ & $-0,631$ & $-0,13$ & 1 & & & \\
\hline PA(C) Loc. & 0,158 & 0,316 & 0,158 & $-0,656$ & $-0,394$ & $-0,158$ & 0,16 & 0 &, $805^{\star}$ & 0,242 & 0,483 & 0,638 & $-0,474$ & $-0,656$ & $-0,316$ & 0 & 0,08 & 1 & & \\
\hline $\mathrm{PA}(\mathrm{C}) \mathrm{MSNV}$ & $-0,144$ & $-0,289$ & $-0,289$ & $-0,3$ & $-0,18$ & 0 & 0,073 & $-0,483$ & $-0,441$ & $-0,294$ & $-0,147$ & $-0,364$ & $-0,433$ & $-0,3$ & $-0,289$ & $-0,72$ & $-0,22$ & $-0,09$ & 1 & \\
\hline
\end{tabular}

*Valores significativos $(p \leq 0,05)$ - coeficiente de Correlação de Spearman; **Valores significativos $(p \leq 0,01)$ - coeficiente de Correlação de Spearman

Legenda: Tx. Pal. = taxa de palavras; Tx. Pp. = taxa de pseudopalavras; Tx. Texto = taxa de texto; Ac. Pal. = acurácia de palavras; Ac. Pp. = acurácia de pseudopalavras; Ac. Texto = acurácia de texto; MFT-BS = memória fonológica de trabalho com palavras de baixa similaridade; MFT-MS = memória fonológica de trabalho com palavras de média similaridade; MFT-AS = memória fonológica de trabalho com palavras de alta similaridade; MFT-ET = memória fonológica de trabalho - escore total; NR Obj.T = tempo de nomeação rápida de objetos; NR Cor.T = tempo de nomeação rápida de cores; NR Obj Er = erros de nomeação rápida de objetos; NR Cor $\mathrm{Er}$ = erros de nomeação rápida de cores; $\mathrm{CF}=$ consciência fonológica; $\mathrm{PA}(\mathrm{C})$ - Loc. = localização da fonte sonora; $\mathrm{PA}(\mathrm{C})$ - MSNV = memória sequencial não verbal; $\mathrm{PA}(\mathrm{C})-\mathrm{MSV}=$ memória sequencial verbal

Apesar das diferenças encontradas, a investigação de correlações dos três grupos mostrou que, quanto melhor a criança lê, melhor ela escreve (Tabelas 2 a 4). O GC escreveu e reconheceu automaticamente as palavras que lhe foram apresentadas, o que explica a ausência de correlação dessas variáveis com a memória fonológica de trabalho. Por outro lado, o GP2 utilizou mais recursos de memória para escrever corretamente itens isolados, enquanto o GP1 não fez uso desse recurso, uma vez que as médias mais baixas de memória de trabalho foram encontradas nos escolares com TF.

Quanto menos tempo o GP1 despendeu na nomeação rápida de objetos, maior o número de palavras que escreveu corretamente. Embora não tenham sido encontradas pesquisas que investigaram correlação entre nomeação rápida e escrita correta de palavras nessa faixa de escolaridade, existem informações sobre correlações entre bons desempenhos de leitura e menores tempos de acesso fonológico ao léxico mental ${ }^{(8,9,13,17)}$.

Ao contrário do que se observou no $\mathrm{GC}$, as correlações encontradas no GP1 entre as tarefas de leitura e o ditado com a consciência fonológica, mostraram que esse grupo, frequentemente, utilizou a rota fonológica para ler e escrever. A literatura indica que esse tipo de desempenho é mais verificado nos anos iniciais da escolarização, ou na presença de transtornos do aprendizado da leitura e da escrita ${ }^{(5,8,13)}$.

O GP1 não apresentou correlações da memória de trabalho para as palavras de baixa similaridade. Esse padrão de ausência de correlações com as tarefas de codificação e decodificação assemelhou-se ao do GC. Porém, é preciso lembrar que o GP1 mostrou médias de acerto muito mais baixas que o GC. Além disso, pode-se pensar que enquanto os escolares de bom rendimento escolar (GC) prescindiram de recursos da memória fonológica para escrever ou reconhecer palavras escritas, os do GP1, sequer a utilizaram, na medida em que sua capacidade era deficitária.

As correlações encontradas no GP1 sugerem que a presença de alterações na fala pode ter influenciado o baixo desempenho 
Tabela 4. Correlações entre as variáveis avaliadas em GP2

\begin{tabular}{|c|c|c|c|c|c|c|c|c|c|c|c|c|c|c|c|c|c|c|c|c|}
\hline & $\begin{array}{l}\dot{\sigma} \\
\dot{\square} \\
\dot{x} \\
\qquad\end{array}$ & $\begin{array}{l}\dot{\alpha} \\
\dot{x} \\
\dot{x}\end{array}$ & 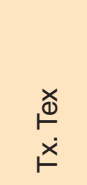 & $\begin{array}{l}\dot{\sigma} \\
0 \\
\dot{0} \\
\dot{\alpha}\end{array}$ & $\begin{array}{l}\frac{\dot{\alpha}}{Q} \\
\dot{0}\end{array}$ & $\begin{array}{l}\underset{\odot}{\oplus} \\
\underset{0}{0}\end{array}$ & 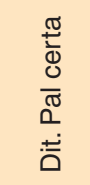 & 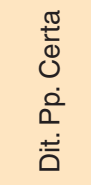 & 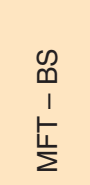 & $\begin{array}{l}\sum_{1}^{\infty} \\
1 \\
5 \\
\end{array}$ & $\begin{array}{l}0 \\
1 \\
1 \\
\stackrel{L}{\Sigma} \\
\Sigma\end{array}$ & $\begin{array}{l}5 \\
1 \\
5 \\
5 \\
\Sigma\end{array}$ & 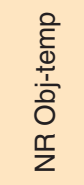 & $\begin{array}{l}\frac{0}{\frac{1}{0}} \\
\frac{1}{0} \\
\frac{1}{0} \\
\frac{\Upsilon}{Z}\end{array}$ & $\begin{array}{l}\stackrel{ }{E} \\
\Phi \\
\frac{ \pm}{1} \\
\frac{1}{0} \\
\mathcal{U} \\
\frac{\Upsilon}{Z}\end{array}$ & 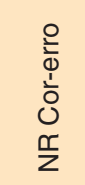 & U & $\frac{\dot{0}}{\frac{0}{0}}$ & 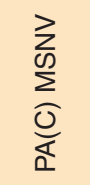 & $\frac{\sum_{0}^{\infty}}{0}$ \\
\hline Tx. Pal. & 1 & & & & & & & & & & & & & & & & & & & \\
\hline Tx. Pp. &, $952^{* *}$ & 1 & & & & & & & & & & & & & & & & & & \\
\hline Ac. Pal. &, $898^{* *}$ &, $850^{* *}$ &, $958^{* *}$ & 1 & & & & & & & & & & & & & & & & \\
\hline Ac. Pp. &, $874^{\star *}$ &, $874^{\star *}$ &, $934^{\star \star}$ &, $976^{\star *}$ & 1 & & & & & & & & & & & & & & & \\
\hline Ac. Tex &, $929^{\star \star}$ &, $881^{\star *}$ &, $976^{\star *}$ &, $994^{* *}$ &, $970^{* *}$ & 1 & & & & & & & & & & & & & & \\
\hline Dit. Pal acert &, $874^{\star \star}$ &, $874^{\star \star}$ &, $934^{\star \star}$ &, $976^{\star *}$ &, $952^{\star *}$ &, $970^{* *}$ & 1 & & & & & & & & & & & & & \\
\hline MFT-AS & 0,051 & 0,18 & 0 & 0,078 & 0,078 & 0,077 & 0,207 & 0,331 & 0,521 & 0,582 & 1 & & & & & & & & & \\
\hline MST-ET & 0,619 & 0,571 & 0,595 & 0,647 & 0,551 & 0,667 & 0,695 & 0,575 &, $952^{\star *}$ &, $994^{\star \star}$ & 0,643 & 1 & & & & & & & & \\
\hline NR Obj- T & $-0,69$ & $-0,524$ & $-0,643$ & $-0,659$ & $-0,563$ & $-0,69$ & $-0,587$ & $-0,434$ &,$- 807^{*}$ &,$- 838^{\star *}$ & $-0,18$ &,$- 810^{\star}$ & 1 & & & & & & & \\
\hline NR Obj-Er & $-0,707$ & $-0,551$ & $-0,671$ & $-0,705$ & $-0,62$ &,$- 731^{*}$ & $-0,633$ & $-0,488$ &,$- 830^{*}$ &,$- 855^{\star \star}$ & $-0,22$ &,$- 826^{*}$ &, $994^{\star *}$ & 1 & & & & & & \\
\hline NRCor-T & 0,311 & 0,491 & 0,287 & 0,133 & 0,205 & 0,204 & 0,241 & 0,283 & 0,152 & 0,06 & 0,013 & 0,06 & 0,072 & 0,096 & 1 & & & & & \\
\hline NR Cor-Er & 0,452 & 0,571 & 0,429 & 0,263 & 0,311 & 0,333 & 0,335 & 0,332 & 0,205 & 0,096 & $-0,18$ & 0,071 & $-0,071$ & $-0,036$ & $958^{\star *}$ & 1 & & & & \\
\hline CF & 0,659 & 0,539 & 0,623 & 0,687 & 0,663 & 0,683 & 0,59 & 0,604 &, $727^{\star}$ &, $711^{*}$ & 0,31 & 0,671 &,$- 802^{*}$ &,$- 849^{\star \star}$ & $-0,349$ & $-0,26$ & 1 & & & \\
\hline PA(C) Loc. & . & . & . & . & . & . & . & . & . & . & . & . & . & . & . & . & . & . & & \\
\hline $\mathrm{PA}(\mathrm{C}) \mathrm{MSNV}$ & 0 & 0 & $-0,096$ & $-0,187$ & $-0,187$ & $-0,096$ & $-0,187$ & $-0,236$ & 0,146 & 0,242 & 0,193 & 0,289 & $-0,385$ & $-0,339$ & 0,436 & 0,385 & 0,021 & . & 1 & \\
\hline
\end{tabular}

*Valores significativos $(p \leq 0,05)$ - coeficiente de Correlação de Spearman; **Valores significativos $(p \leq 0,01)$ - coeficiente de Correlação de Spearman

Legenda: Tx. Pal. $=$ taxa de palavras; Tx. Pp. = taxa de pseudopalavras; Tx. Texto = taxa de texto; Ac. Pal. $=$ acurácia de palavras; Ac. Pp. $=$ acurácia de pseudopalavras; Ac. Texto = acurácia de texto; MFT-BS = memória fonológica de trabalho com palavras de baixa similaridade; MFT-MS = memória fonológica de trabalho com palavras de média similaridade; MFT-AS = memória fonológica de trabalho com palavras de alta similaridade; MFT-ET = memória fonológica de trabalho - escore total; NR Obj.T = tempo de nomeação rápida de objetos; NR Cor.T = tempo de nomeação rápida de cores; NR Obj Er = erros de nomeação rápida de objetos; NR Cor $\mathrm{Er}$ = erros de nomeação rápida de cores; $\mathrm{CF}=$ consciência fonológica; $\mathrm{PA}(\mathrm{C})-$ Loc. = localização da fonte sonora; $\mathrm{PA}(\mathrm{C})-\mathrm{MSNV}=$ memória sequencial não verbal; $\mathrm{PA}(\mathrm{C})-\mathrm{MSV}=$ memória sequencial verbal

desse grupo em $\mathrm{CF}$, que, consequentemente, produziu maior número de erros na leitura e na escrita. Algumas discordâncias encontradas na literatura ${ }^{(14)}$ são, provavelmente, fruto de estudos que avaliaram crianças de $1^{a}$ série, em fase de alfabetização, com o desenvolvimento da consciência fonológica ainda em processo.

Somente no GP1 verificaram-se correlações do processamento auditivo com o acesso fonológico ao léxico mental e à memória fonológica de trabalho. Considerando que a tarefa do processamento auditivo que se correlacionou com outras do processamento fonológico tem características semelhantes à da prova de memória fonológica de curto prazo, as correlações podem ser entendidas pela natureza da tarefa e pela forte dependência de ambas com a organização do sistema fonológico.

Os recursos de memória fonológica de trabalho apresentados pelo GP2 foram utilizados para a escrita correta de itens isolados e para a leitura com velocidade e precisão adequadas. Ou seja, quanto melhor a memória, melhor o desempenho em leitura e escrita, a despeito de terem mostrado baixos valores de parâmetros de fluência leitora e de precisão de escrita. Essas associações entre a memória de trabalho e as tarefas de leitura e de escrita demonstram que esses escolares ainda não alcançaram a rota lexical de leitura ${ }^{(8,12,13)}$.

Observou-se, no GP2 e nos demais grupos, que a memória de trabalho para pseudopalavras de baixa e média similaridade correlacionou-se positivamente com o escore total da prova. Deve-se ressaltar que nem todos os escolares com TLE apresentam as mesmas habilidades para processar a informação fonológica $^{(8,13,16,17)}$. No GP2, a CF correlacionou-se positivamente com a memória para pseudopalavras de baixa e média similaridade. Esses resultados confirmam outros da literatura, sobre a estruturação do processamento fonológico, composto pela consciência fonológica, memória fonológica de trabalho e acesso fonológico ao léxico mental: intimamente relacionados, formam a base para o aprendizado do princípio alfabético ${ }^{(5,23)}$.

Considerando os resultados dos demais grupos, no que diz respeito ao processamento fonológico e auditivo, o GP2 
apresentou desempenho intermediário e conseguiu desenvolver estratégias que, provavelmente, favoreceram esse resultado, apesar de mantê-lo na rota fonológica de leitura e escrita.

Quanto às habilidades do processamento auditivo, o GP2 mostrou que apenas a memória sequencial verbal correlacionou-se às taxas (itens isolados) e às acurácias de leitura, ao ditado de palavras e à consciência fonológica. É provável que essas correlações estejam ligadas, principalmente, à linguagem e seus transtornos, sendo por isso mais encontradas no GP2 ${ }^{(28-30)}$.

Portanto, pode-se pensar que os padrões de correlação encontrados indicaram ser pertinentes à existência de relação de continuidade entre linguagem oral e fala e leitura e escrita. Essa relação evidencia a importância da investigação e da avaliação, pelo fonoaudiólogo, dos processamentos e habilidades subjacentes às manifestações de leitura e escrita que podem estar, também, na base de alterações de fala e linguagem oral. Consequentemente, mostram a necessidade de se identificar o nível funcional em que esses déficits estão presentes e o tipo de relações que mantêm entre si, provavelmente fortes o suficiente para deflagrar prejuízos no aprendizado escolar.

É possível fazer essas afirmações na medida em que, nesta pesquisa, os escolares do GP1 mostraram, estatisticamente, mais baixos valores nas tarefas de taxa e acurácia de leitura, ditado, CF, memória fonológica (palavras de baixa e média similaridade e escore total) e memória sequencial verbal.

O tamanho da amostra revelou-se limitado, todavia, para o alcance de dados que pudessem evidenciar de forma mais robusta as diferenças encontradas entre os três grupos estudados e, principalmente, para diferenciar os dois grupos com TLE. Os critérios estabelecidos para inclusão e exclusão da amostra e o baixo número de TCLE assinados, certamente interferiram no número amostral.

\section{CONCLUSÃO}

Os diferentes padrões de correlações encontrados para cada grupo podem confirmar as diferenças observadas entre os grupos que apresentaram o pior desempenho do grupo com TLE e TF, principalmente em tarefas que demandaram informações da organização fonológica. As correlações do GC evidenciam capacidades de reconhecimento automático de palavras e de escrita ortográfica. As correlações encontradas na análise do GP2 mostram que os escolares com TLE, mas sem TF, utilizaram recursos do processamento fonológico, sobretudo da memória, para ler mais rapidamente e com mais precisão e, da mesma forma, escrever melhor e mais corretamente. O GP1, por outro lado, desprovido de melhores condições de processamento fonológico, principalmente as de memória fonológica, mostrou os piores desempenhos e mais baixos valores de taxa e acurácia de leitura e de acertos na escrita de palavras e pseudopalavras.

Os padrões de correlação, associados aos valores mais baixos de respostas podem mostrar a influência dos transtornos fonológicos sobre o pior desempenho em leitura e escrita.

\section{REFERÊNCIAS}

1. Webster PE, Plante AS. Effects of phonological impairment on word, syllable, and phoneme segmentation and reading. Lang Speech Hear Serv Sch. 1992;23:176-82.

2. Pascoe M, Satackhouse J, Wells B. Persisting speech difficulties in children: children`s speech and literacy difficulties 3. Sussex: Whurr Publishers Limited, 2006.

3. Pennington BF, Bishop DVM. Relations among speech, language and reading disorders. Annu Rev Psychol. 2009;60:283-306.

4. Ritter MJ. The speech-language pathologist and reading: opportunities to extend services for the children we serve. Perspectives on School-Based Issues. 2009;10(2):38-44.

5. Snowling MJ, Hulme C. Evidence-based interventions for reading and language difficulties: creating a virtuous circle. Br J Educ Psychol. 2011;81:1-23.

6. Patah LK, Takiuchi N. Prevalência das alterações fonológicas e uso dos processos fonológicos em escolares aos 7 anos. Rev CEFAC. 2008;10(2):158-67.

7. Rabelo ATV, Alves CRL, Goulart LMHF, Friche AAL, Lemos SMA, Campos FR, et al. Alterações de fala em escolares na cidade de Belo Horizonte. J Soc Bras Fonoaudiol. 2011;23(4):344-50.

8. Salgado C, Capellini SA. Desempenho em leitura e escrita de escolares com transtorno fonológico. Psicol Esc Educ. 2004;8(2):179-88.

9. Johnson EP, Pennington BF, Lowenstein JH, Nittrouer S. Sensitivity to structure in the speech signal by children with speech sound disorder and reading disability. J Comm Disord. 2011;44:294-314.

10. Silva MC, Souza AF, Chang EM, Beltrame JM, Pinto JCBR, Ávila CRB. Influência das alterações de fala sobre a escrita. In: XVIII Congresso Brasileiro de Fonoaudiologia; 2010. set 22-25. Curitiba. Anais. Congresso Brasileiro de Fonoaudiologia; Curitiba: 2010. p.3017.

11. Arnaut MA, Beltrame JM, Chang EM, Nepomuceno P, Tizzi PM, Ávila CRB. Associação entre processamento auditivo, processamento fonológico e vocabulário em escolares com transtornos da leitura e da escrita. In: XX Congresso Brasileiro de Fonoaudiologia; 2012. Out 31- Nov 02. Brasília. Anais. Congresso Brasileiro de Fonoaudiologia; Curitiba: 2012. p.2903.

12. Jones G, Tamburelli M, Watson SE, Gobet F, Pine JM. Lexicality and frequency in specific language impairment: accuracy and error data from two nonword repetition tests. J Speech Lang Hear Res. 2010;53:1642-55.

13. Capellini SA, Conrado TLBC. Desempenho de escolares com e sem dificuldades de aprendizagem de ensino particular em habilidade fonológica, nomeação rápida, leitura e escrita. Rev CEFAC. 2009;11(Suppl.2):183-93.

14. Rizzon GF, Chiechelski P, Gomes E. Relação entre consciência fonológica e desvio fonológico em crianças da $1^{\mathrm{a}}$ série do ensino fundamental. Rev CEFAC. 2009;11(Suppl.2):201-7.

15. Donicht G, Nazari GT. Relação entre desvios fonológicos e consciência fonológica. Letrônica. 2010;3(1):22-41. 
16. Cardoso MH, Romero ACL, Capellini SA. Alterações dos processos fonológicos e índice de gravidade entre escolares com dislexia e escolares com bom desempenho acadêmico. Rev Soc Bras Fonoaudiol. 2012;17(3):287-92.

17. Bicalho LGR, Alves LM. A nomeação seriada rápida em escolares com e sem queixas de problemas de aprendizagem em escola pública e particular. Rev. CEFAC. 2010;12(4):608-16.

18. Ferreiro E, Teberosky A. Psicogênese da língua escrita. 4a. ed. Porto Alegre: Artes Médicas; 1999.

19. Pereira LD, Schochat E. Processamento auditivo central: manual de avaliação. São Paulo: Lovise; 1997.

20. Carvalho CAF, Ávila CRB, Chiari BM. Níveis de compreensão de leitura em escolares. Pro-Fono. 2009;21(3):207-12.

21. Kida ASB, Chiari BM, Ávila CRB. Escalas de avaliação da leitura e da escrita: evidências preliminares de confiabilidade. Pro-Fono. 2010;22(4):509-14.

22. Saraiva RA, Moojen SMP e Murarki R. Avaliação da compreensão leitora de textos expositivos. São Paulo: Casa do Psicólogo, 2006.

23. Paolucci JF, Ávila CRB. Competência ortográfica e metafonológica: influências e correlações na leitura e escrita de escolares da $4^{\mathrm{a}}$ série. Rev Soc Bras Fonoaudiol. 2009;14(1):48-55.
24. Andrade CRF, Befi-Lopes DM, Fernandes FDM, Wertzner HF, ABFW - Teste de linguagem infantil, nas áreas de fonologia vocabulário, fluência e pragmática. 2a. ed. Carapicuiba: Pró-Fono; 2004. 116p.

25. Santos FH, Bueno OFA. Validation of the Brazilian children's test of pseudoword repetition in Portuguese speakers aged 4 to 10 years. Braz J Med Biol Res. 2003;36(11):1533-47.

26. Wagner RK, Torgesen JK, Rashotte CA. CTOPP Comprehensive Test of Phonological Processing. Austin: Pro-Ed, 1999.

27. Capovilla AGS, Capovilla FC. Prova de consciência fonológica: desenvolvimento de dez habilidades da pré-escola à segunda série. Temas Desenvolv. 1998;7(37):14-20.

28. Engelmann L, Ferreira MIDC. Avaliação do processamento auditivo em crianças com dificuldades de aprendizagem. Rev Soc Bras Fonoaudiol. 2009;14(1):69-74.

29. Caumo DTM, Fereira MIDC. Relação entre desvios fonológicos e processamento auditivo. Rev Soc Bras Fonoaudiol. 2009;14(2):23440.

30. Pelitero TM, Manfredi AKS, Schneck APC. Avaliação das habilidades auditivas em crianças com alterações de aprendizagem. Rev CEFAC. 2010;12(4):662-70. 